\title{
Image-Guided Neutron Capture Therapy Using the Gd-DO3A-BTA Complex as a New Combinatorial Treatment Approach
}

\author{
Ki-Hye Jung $\left(\mathbb{D},{ }^{1}\right.$ Ji-Ae Park $\left(\mathbb{D},{ }^{1}\right.$ Jung Young Kim $\left(\mathbb{D},{ }^{1}\right.$ Mi Hyun Kim, ${ }^{1}$ Seyoung Oh, \\ Hee-Kyung Kim, ${ }^{2}$ Eun-Ji Choi, ${ }^{3}$ Han-Jun Kim, ${ }^{3}$ Sun Hee Do ${ }^{D},{ }^{3}$ Kyo Chul Lee, ${ }^{1}$ \\ Kyeong Min Kim $\mathbb{D}$, ${ }^{1}$ Yong Jin Lee $\mathbb{D}^{1},{ }^{1}$ and Yongmin Chang $\mathbb{D}^{4,5}$ \\ ${ }^{1}$ Division of Applied RI, Korea Institute of Radiological and Medical Science, Seoul 139-706, Republic of Korea \\ ${ }^{2}$ BK21 Plus KNU Biomedical Convergence Program, School of Medicine, Kyungpook National University, Daegu 700-721, \\ Republic of Korea \\ ${ }^{3}$ College of Veterinary Medicine, Konkuk University, Seoul 05029, Republic of Korea \\ ${ }^{4}$ Department of Molecular Medicine, School of Medicine, Kyungpook National University, Daegu 700-721, Republic of Korea \\ ${ }^{5}$ Department of Radiology, Kyungpook National University Hospital, Daegu 700-721, Republic of Korea
}

Correspondence should be addressed to Yong Jin Lee; yjlee@kirams.re.kr and Yongmin Chang; ychang@knu.ac.kr

Received 4 June 2018; Revised 17 August 2018; Accepted 30 September 2018; Published 1 November 2018

Guest Editor: Charalampos Tsoumpas

Copyright (c) $2018 \mathrm{Ki}$-Hye Jung et al. This is an open access article distributed under the Creative Commons Attribution License, which permits unrestricted use, distribution, and reproduction in any medium, provided the original work is properly cited.

Gadolinium-neutron capture therapy (Gd-NCT) is based on the nuclear capture reaction that occurs when ${ }^{157}$ Gd is irradiated with low energy thermal neutrons to primarily produce gamma photons. Herein, we investigated the effect of neutron capture therapy (NCT) using a small molecular gadolinium complex, Gd-DO3A-benzothiazole (Gd-DO3A-BTA), which could be a good candidate for use as an NCT drug due to its ability to enter the intracellular nuclei of tumor cells. Furthermore, MRI images of Gd-DO3A-BTA showed a clear signal enhancement in the tumor, and the images also played a key role in planning NCT by providing accurate information on the in vivo uptake time and duration of Gd-DO3A-BTA. We injected Gd-DO3A-BTA into MDA-MB-231 breast tumor-bearing mice and irradiated the tumors with cyclotron neutrons at the maximum accumulation time (postinjection $6 \mathrm{~h}$ ); then, we observed the size of the growing tumor for 60 days. Gd-DO3A-BTA showed good therapeutic effects of chemo-Gd-NCT for the in vivo tumor models. Simultaneously, the Gd-DO3A-BTA groups ([Gd-DO3A-BTA(+), NCT(+)]) showed a significant reduction in tumor size $(p<0.05)$, and the inhibitory effect on tumor growth was exhibited in the following order: [Gd-DO3A-BTA $(+), \mathrm{NCT}(+)]$ $>[$ Gd-DO3A-BTA $(+)$, NCT $(-)]>[$ Gd-DO3A-BTA $(-), \mathrm{NCT}(+)]>[$ Gd-DO3A-BTA $(-)$, NCT $(-)]$. On day 60, the [Gd-DO3A-BTA $(+), \mathrm{NCT}(+)]$ and $[\mathrm{Gd}-\mathrm{DO} 3 \mathrm{~A}-\mathrm{BTA}(-), \mathrm{NCT}(-)]$ groups exhibited an approximately 4.5 -fold difference in tumor size. Immunohistochemistry studies demonstrated that new combinational therapy with chemo-Gd-NCT could treat breast cancer by both the inhibition of tumor cell proliferation and induction of apoptosis-related proteins, with in vivo tumor monitoring by MRI.

\section{Introduction}

Neutron capture therapy (NCT) is a well-known approach to cancer treatment based on the accumulation of neutron capture agents at the tumor site [1], followed by irradiation with thermal neutrons. NCT is a very effective technique for cancer treatment because the thermal neutrons with low energy do not cause damage to normal cells that lack the neutron capture compounds. This technique thus provides a solution to the major problem of radiation therapy, which is the radiation-induced damage to normal tissue. Therefore, the strategy for the accumulation of NCT compounds specifically within the tumor is critical to avoid damage to normal tissues [2-5].

Gadolinium $\left({ }^{157} \mathrm{Gd}\right)$-based NCT (Gd-NCT) has generated recent interest as a cancer treatment due to the 
following merits. (i) The ${ }^{157} \mathrm{Gd}$ atom captures neutrons $\left({ }^{157} \mathrm{Gd}+\mathrm{n}_{\text {th }}(0.025 \mathrm{eV}) \longrightarrow\left[{ }^{158} \mathrm{Gd}\right] \longrightarrow{ }^{158} \mathrm{Gd}+\gamma+\right.$ $7.94 \mathrm{MeV})$ across a very large cross section $\left(\sigma_{\mathrm{th}}=240,000 \mathrm{~b}\right)$. (ii) The kinetic energy of Gd-NCT, with a mixture of low- and high-energy ionizing particles, is more uniformly distributed throughout tumor tissues, and can be used to solve the shortcomings of heterogeneous in vivo dose distribution. (iii) ${ }^{157} \mathrm{Gd}$ complexes are widely developed as magnetic resonance imaging (MRI) contrast agents by chelation chemistry and have been routinely used in clinical applications including Gadovist ${ }^{\circledR}$ (gadobutrol), Dotarem ${ }^{\circledR}$ (gadoterate meglumine) and Omniscan ${ }^{\circledR}$ (gadodiamide) [6]. Gadolinium MRI contrast agents could thus be first considered as NCT agents, but it is difficult to specifically target tumor cells in vivo. These agents do not accumulate well in tumor tissue during neutron irradiation after an intravenous injection for therapy, as shown in Table 1. The data show high uptake of the contrast agents into tumor cells at 5 mins, but they exhibit very low uptake at 2 hrs. To obtain success with ${ }^{157} \mathrm{Gd}-\mathrm{NCT},{ }^{157} \mathrm{Gd}$ must be transferred into tumor cells at high concentrations during neutron irradiation. In a previous study, a $50-200 \mu \mathrm{g}{ }^{157} \mathrm{Gd} / \mathrm{g}$ tumor was reported as an effective cancer treatment.

For the ${ }^{157} \mathrm{Gd}$ neutron capture reaction, the majority of the energy is released as long-range gamma radiation, while, $0.63 \%$ of the time, this emission occurs as Auger and conversion electrons. Auger electrons generated from GdNCT have strong in vivo cytotoxicity by high linear energy transfer (LET), which can induce DNA double-strand breaks (DSB) and restrain the proliferation of tumor cells. Thus, increase in the therapeutic effects is observed when Gd atoms are highly internalized into tumor cells $[18,19]$.

As therapeutic candidates, various benzothiazoles deserve special attention, as they are known to possess diverse biological properties such as anti-inflammatory, antimicrobial, and anticancer effects. Some of the compounds containing the benzothiazole system are in clinical usage for the treatment of various diseases/disorders [20]. In our previous study, the complexes have not only displayed tumor specificity but also enhanced intracellular MR images of the cytosol and nuclei of a series of tumor cells. The antiproliferative activity of Gd-DO3A-BTA (Chart 1), which contains a chelating moiety (DO3A) and a chemoagent region (BTA), was demonstrated by determining the in vitro growth inhibition values $\left(\mathrm{GI}_{50}\right.$ and $\left.\mathrm{TGI}\right)$ and monitoring tumor volume regression in vivo. In particular, Gd-DO3ABTA has been reported to specifically accumulate intracellularly in tumors arising from MDA-MB-231 breast cancer cells $[9,21]$. On the basis of this result, we make attempts to treat in vivo tumor tissue by neutron beam irradiation with a medical cyclotron, preserving the high tumor uptake of ${ }^{157} \mathrm{Gd}$ complexes.

\section{Results}

2.1. In Vivo MR Imaging. Figure 1(a) shows the in vivo T1weighted MR images of mice that were injected with GdDO3A-BTA $(0.1 \mathrm{mmol} \mathrm{Gd} / \mathrm{kg})$ via the tail vein. T1-weighted MR images were used because the T1 shortening effect is more dominant at the relatively low Gd concentrations used $(\sim 0.1 \mathrm{mmol} \mathrm{Gd} / \mathrm{kg})$. The MR image reveals clear tumor enhancement, which increased for $6 \mathrm{~h}$ before gradually decreasing (Figure 1(b)). This result is consistent with the biodistribution data reported in our previous study [9], demonstrating that we can effectively define the starting point of NCT with in vivo MR imaging.

\subsection{Gd-NCT Inhibited Tumor Growth with Gd-DO3A-BTA.} A small animal study was performed in which mice were irradiated with a neutron beam at $6 \mathrm{~h}$ p.i. to determine the optimal time at which the highest uptake of Gd-DO3A-BTA into the tumor tissue occurred. All other tissues, except the tumor tissues, were covered with a plastic box of Teflon to protect them from irregular showering with neutron beams. Figure 2(a) shows the time-course of change in tumor volume after irradiation with neutron beam for 60 days. The Gd-DO3A-BTA-injected and neutron-irradiated mice group ([Gd-DO3A-BTA $(+), \mathrm{NCT}(+)])$ showed a significant decrease in tumor size than the nontreated groups ([GdDO3A-BTA $(+)$, NCT(-)], [Gd-DO3A-BTA(-), NCT(+)], and $[\mathrm{Gd}-\mathrm{DO} 3 \mathrm{~A}-\mathrm{BTA}(-), \mathrm{NCT}(-)])$. Four groups began to show significant changes in tumor volume on day 15 . In particular, the difference between [Gd-DO3A-BTA $(+)$, $\mathrm{NCT}(+)]$ and nontreated groups on days 42,50 , and 60 was significant $(p<0.05)$. The tumor growth suppression was observed in the following order: [Gd-DO3A-BTA(+), NCT $(+)]>[\mathrm{Gd}-\mathrm{DO} 3 \mathrm{~A}-\mathrm{BTA}(+), \mathrm{NCT}(-)]>[\mathrm{Gd}-\mathrm{DO} 3 \mathrm{~A}-\mathrm{BTA}(-)$, $\mathrm{NCT}(+)]>[\mathrm{Gd}-\mathrm{DO} 3 \mathrm{~A}-\mathrm{BTA}(-), \mathrm{NCT}(-)]$. On day 60 , [GdDO3A-BTA $(+), \operatorname{NCT}(+)]$ and [Gd-DO3A-BTA(-), NCT(-)] groups showed an approximately 4.5 -fold difference in tumor size. The mean tumor volume (relative tumor volume) of the [Gd-DO3A-BTA(+), NCT(+)] group was $11.99 \pm 5.05$, which was much smaller than that in the other groups $(p<0.05)$ on day 60. Due to the tumor suppressive effects of BTA, the antitumor activity of Gd-DO3A-BTA alone (no neutron beam irradiation) was higher than that of neutron irradiation alone. Consistent with earlier reports [10, 22, 23], the neutron irradiation [Gd-DO3A-BTA(-), NCT(+)] alone group also showed some inhibition of tumor growth than the group that did not receive either treatment [Gd-DO3A-BTA(-), $\mathrm{NCT}(-)$ ]. After irradiation by neutron beams, changes in the body weight of the mice were measured as well as tumor volume. As shown in Figure 2(b), no significant weight loss was observed, suggesting the safety of Gd-DO3A-BTA for NCT. The suppression of tumor growth could be clearly seen from the MR images and morphological findings shown in Figure 3.

2.3. Gd-NCT Inhibited Tumor Cell Proliferation and Induced Tumor Cell Apoptosis with Gd-DO3A-BTA. The in vivo antitumor activity of Gd-DO3A-BTA in NCT was further verified with histological evaluation and immunohistochemistry, as shown in Figure 4. H\&E staining of tumor sections showed proliferating tumor cells and necrotic regions. There were no apparent differences in growth patterns and areas of necrosis between groups. To assess the effect of Gd-NCT on tumor suppression and apoptosis, immunohistochemistry for Ki-67, cleaved caspase 3, and caspase 8, as well as TUNEL staining, was performed. The expression of 
TABLE 1: Various types of gadolinium agents for NCT.

\begin{tabular}{|c|c|c|c|c|c|c|}
\hline & Form & $\begin{array}{l}\text { Injection } \\
\text { route }\end{array}$ & $\begin{array}{l}\text { Injection amount } \\
(\mathrm{Gd})\end{array}$ & $\begin{array}{c}\text { Tumor accumulation } \\
\text { Gd/g tumor }\end{array}$ & Highest uptake time (ICP time) & Reference \\
\hline \multirow{4}{*}{ Low molecules } & Gd-DTPA & i.t. & $1200 \mu \mathrm{g} /$ mouse & $\begin{array}{l}451 \mu \mathrm{g} / \mathrm{g} \\
5.3 \mu \mathrm{g} / \mathrm{g}\end{array}$ & $\begin{array}{c}5 \mathrm{~min} \\
24 \mathrm{~h}\end{array}$ & [7] \\
\hline & Gd-DTPA & i.v. & $0.1 \mathrm{mmol} / \mathrm{kg}$ & $\begin{array}{l}70.39 \pm 8.75 \mu \mathrm{g} / \mathrm{g} \\
30.22 \pm 4.91 \mu \mathrm{g} / \mathrm{g}\end{array}$ & $\begin{array}{c}5 \mathrm{~min} \\
2 \mathrm{~h}\end{array}$ & [8] \\
\hline & Gd-BOPTA & i.v. & $0.1 \mathrm{mmol} / \mathrm{kg}$ & $\begin{array}{c}100.33 \pm 7.91 \mu \mathrm{g} / \mathrm{g} \\
40.93 \pm 1.83 \mu \mathrm{g} / \mathrm{g}\end{array}$ & $\begin{array}{c}5 \mathrm{~min} \\
2 \mathrm{~h}\end{array}$ & {$[8]$} \\
\hline & $\begin{array}{c}\text { Gd-DO3A- } \\
\text { BTA }\end{array}$ & i.v. & $0.1 \mathrm{mmol} / \mathrm{kg}$ & $221 \mu \mathrm{g} / \mathrm{g}$ & $6 \mathrm{~h}$ & {$[9]$} \\
\hline \multirow{7}{*}{ Nanoparticles } & Liposome & i.v. & $\mathrm{N} / \mathrm{D}$ & $40.277 \pm 2.512 \mu \mathrm{g} / \mathrm{g}$ & $2 \mathrm{~h}$ & {$[10]$} \\
\hline & Liposome & i.v. & $20 \mathrm{mg} / \mathrm{kg}$ & $158.8 \pm 115.6 \mu \mathrm{g} / \mathrm{g}$ & $12 \mathrm{~h}$ & {$[11,12]$} \\
\hline & Lipid emulsions & i.p. & $6 \mathrm{mg} /$ hamster & $107 \mu \mathrm{g} / \mathrm{g}$ & $48 \mathrm{~h}$ & {$[13]$} \\
\hline & Micelles & i.v. & $0.02 \mathrm{mmol} / \mathrm{kg}$ & $3.9 \% \mathrm{ID} / \mathrm{g}^{\mathrm{a}}$ & $10 \mathrm{~h}$ & {$[14]$} \\
\hline & Lipid NPs & i.v. & $6 \mathrm{mg} /$ hamster & $100.7 \mu \mathrm{g} / \mathrm{g}$ & $12 \mathrm{~h}$ & [15] \\
\hline & Chitosan NPs & i.t. & $2.4 \mathrm{mg} / \mathrm{mouse}^{\mathrm{b}}$ & $1766 \pm 96 \mu \mathrm{g} /$ tumor tissue ${ }^{c}$ & $8 \mathrm{~h}$ & {$[16,17]$} \\
\hline & Chitosan NPs & i.t. & $1200 \mu \mathrm{g} /$ mouse & $897.1 \mu \mathrm{g} / \mathrm{g}$ & $24 \mathrm{~h}$ & [7] \\
\hline
\end{tabular}

N/D: not determined; i.t.: intratumoral; i.v.: intravenous; i.p.: intraperitoneal. ${ }^{a} \% \mathrm{ID} / \mathrm{g}$ : percentages of the total injected dose per organ weight. ${ }^{b}$ Administered twice by i.t. injection $24 \mathrm{~h}$ and $8 \mathrm{~h}$ before the assay. ${ }^{\circ}$ The $\mathrm{Gd}$ content in melanoma tissue in mice.

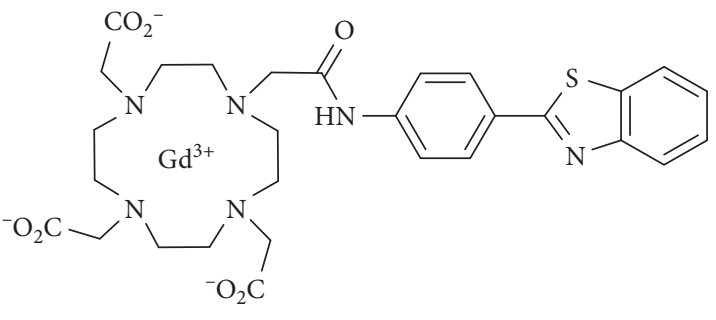

Chart 1: Structure of Gd-DO3A-BTA.

the proliferation marker Ki-67 was significantly decreased in the [Gd-DO3A-BTA $(+)$, NCT(+)] group compared to that of the [Gd-DO3A-BTA(-), NCT(-)] group. All irradiated groups including [Gd-DO3A-BTA $(+), \mathrm{NCT}(+)]$ and [GdDO3A-BTA(-), NCT(+)] showed a decrease in Ki-67 expression, indicating that the neutron beam irradiation has tumor suppressive effects. In addition, the [Gd-DO3ABTA(+), NCT(-)] group also showed decreased Ki-67 expression, implying the antitumor activity of BTA without neutron beam irradiation. Caspase 8 acts as an initiator of apoptosis and is involved in the extrinsic pathway, whereas caspase 3 acts as an effector of apoptosis [24]. The expression of cleaved caspase 3 and 8 did not show significant differences between groups. However, the expressions of both cleaved caspase 3 and 8 were slightly increased in the irradiated groups [Gd-DO3A-BTA $(+)$, $\mathrm{NCT}(+)]$ and [Gd-DO3A-BTA(-), NCT(+)] compared to that in the nonirradiated [Gd-DO3A-BTA $(+)$, NCT(-)] and [Gd-DO3A-BTA(-), NCT(-)] groups. Moreover, the evaluation of apoptotic cell death by TUNEL staining revealed that the percentage of cells undergoing apoptosis was greater in all treated groups, including the [GdDO3A-BTA $(+), \operatorname{NCT}(+)$ ] group. These results correlate with the finding that the [Gd-DO3A-BTA $(+), \mathrm{NCT}(+)]$ group had the smallest tumor volumes, suggesting that the reduced Ki-67 activity and increased apoptotic cell death after BTA injection and neutron beam irradiation induced tumor growth suppression.

\section{Discussion}

For the success of Gd-NCT as a therapeutic modality for cancer, it is important to effectively deliver and sufficiently accumulate $\mathrm{Gd}$ into tumors. For an effective in vivo GdNCT, an optimal dose of 50-200 $\mu \mathrm{g} \cdot \mathrm{Gd} / \mathrm{g}$ tumor tissue has been reported $[11,25]$. As shown in Table $1,0.1 \mathrm{mmol} / \mathrm{kg}$ of Gd-DO3A-BTA administered as an intravenous injection resulted in approximately $221 \mu \mathrm{g}$ of $\mathrm{Gd} / \mathrm{g}$ tumor tissue [9]. This uptake was approximately 1.4 times higher than that reported previously $(158 \mu \mathrm{g} \cdot \mathrm{Gd} / \mathrm{g}$ tumor tissue) with an intravenous injection of Gd-DTPA-encapsulated liposomes $[11,12]$.

Various nano-based formulations have been developed to deliver sufficient amounts of Gd into tumor cells for GdNCT, and these include nanoparticles [15-17, 26-28], liposomes $[10,11,12]$, emulsions [13, 29], and microcapsules $[7,30]$. Most of these particles were designed to be approximately $100 \mathrm{~nm}$ in size to avoid high uptake by the reticuloendothelial system (RES). However, these particles have limited specific tumor targeting ability, since their delivery completely depends on the enhanced permeability and retention (EPR) effect around tumor sites [11, 14, 31]. Furthermore, these nanoparticles often show high in vivo instability by releasing Gd from the nano-based formulations due to the high solubility of Gd-DTPA in an aqueous solution $[11,13,15]$. Therefore, some nano-based formulations for Gd-NCT use a lipophilic complex such as GdDPTA/pLL or Gd-acetylacetonate (Gd-acac) to prevent the possible release of Gd $[11,29]$. However, as shown in Table 1, these nano-based formulations can be injected only by intraperitoneal or intratumoral methods and cannot be administered intravenously.

Gd-DO3A-BTA, however, is readily administered intravenously. Furthermore, Gd-DO3A-BTA has a relatively 


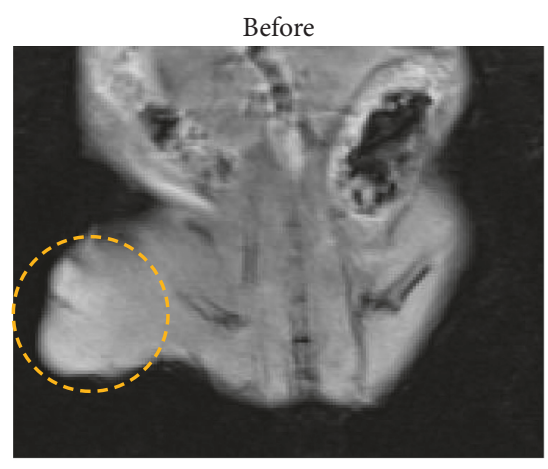

$6 \mathrm{~h}$
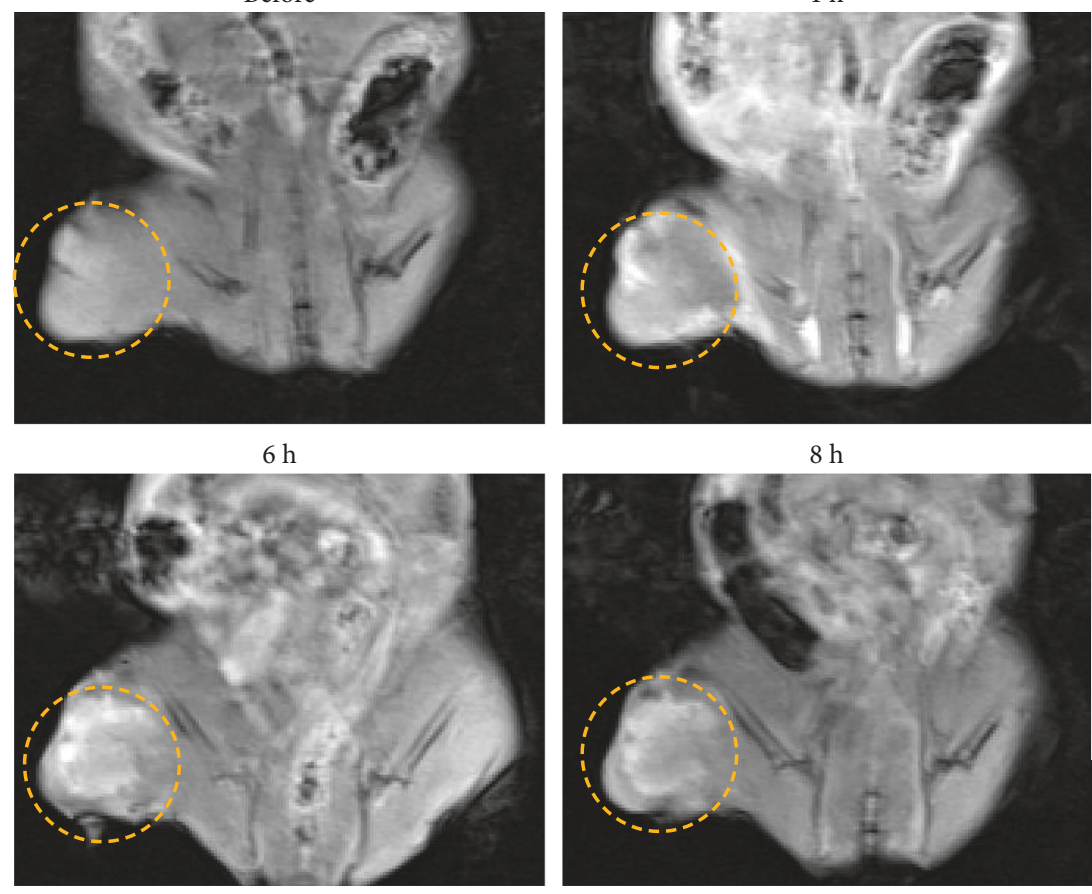

$8 \mathrm{~h}$

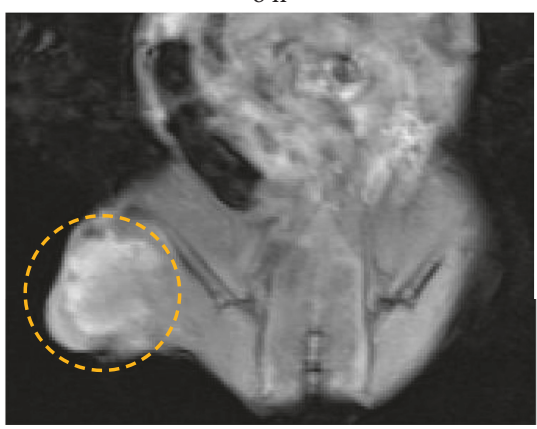

(a)

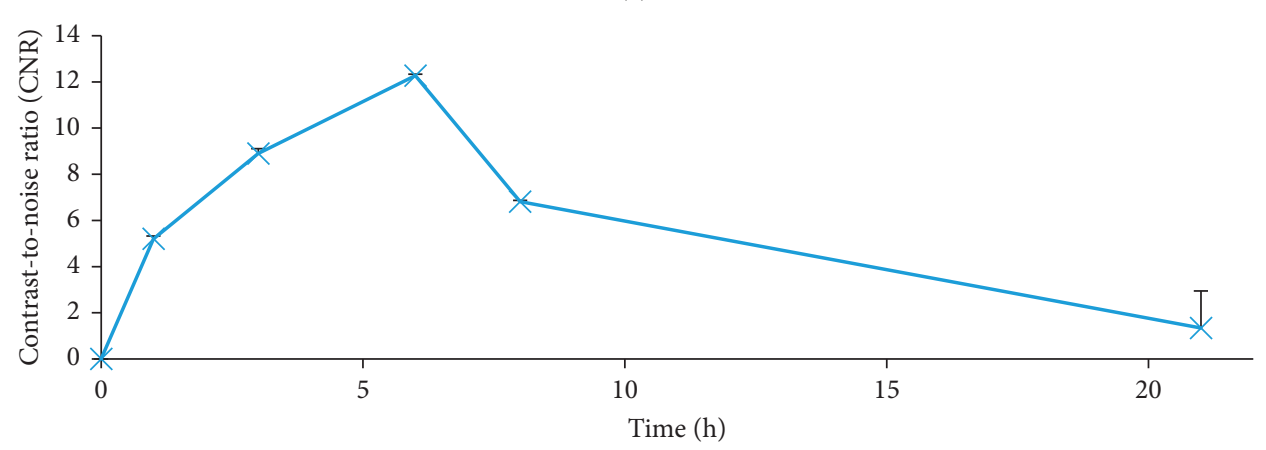

(b)
$3 \mathrm{~h}$

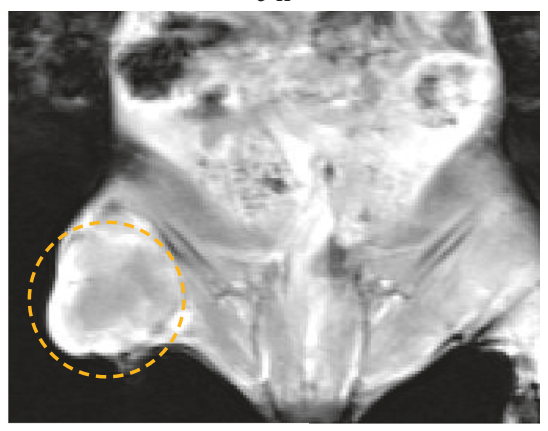

$21 \mathrm{~h}$

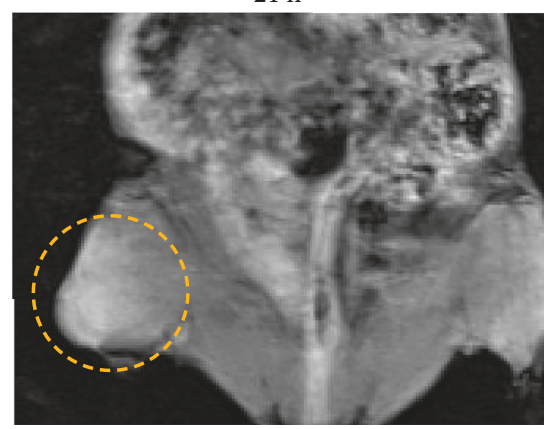

Figure 1: (a) In vivo MR images of nude mice with MDA-MB-231 tumors after intravenous injection of Gd-DO3A-BTA. Tumors are indicated with circles. (b) Contrast-to-noise ratio (CNR) as a function of time.

small molecular weight (M.W. 767) and high chemical stability, which makes it a better tumor-theranostic agent for MR image-guided NCT with a good therapeutic dosage for clinical use. However, for the success of Gd-NCT, Gd must be transferred into tumors at high concentrations during neutron irradiation. The MR imaging data demonstrated that Gd-DO3A-BTA preferentially accumulated in tumor tissue in vivo. Gd-DO3A-BTA delivers more Gd to tumor tissue than conventional Gd-based MRI contrast agents, which may result in a more efficient uptake by the tumor. The effective tumor ablation by Gd-DO3A-BTA could therefore be associated with the high accumulation of Gd in tumor tissue, which in turn captures sufficient thermal neutrons to kill the tumor. Although enriched ${ }^{157} \mathrm{Gd}$ compound would be preferable for Gd-NCT, in this experiment, we utilized the natural Gd compound. The natural abundance of ${ }^{157} \mathrm{Gd}$ is only $15.65 \%$, while enriched compounds may contain up to $90 \%{ }^{157} \mathrm{Gd}$ [20]. Therefore, in the near future, it would be important to perform Gd-NCT experiment with enriched ${ }^{157} \mathrm{Gd}$ compounds to confirm the current results using the natural Gd compound. The tumors in mice that were irradiated and injected with Gd-DO3ABTA were much smaller than those observed in other groups, demonstrating that the Gd-DO3A-BTA could significantly enhance the therapeutic effect of Gd-NCT. In addition, the tumor-therapeutic effects of Gd-DO3A-BTA in combination with NCT were confirmed by MR imaging and histological evaluation. Furthermore, the tumor suppressive effects were evident even after 60 days, suggesting that early treatment of the tumor with a Gd-DO3A-BTA injection, and NCT has the potential to suppress further tumor progression. However, the limitation of the current study should be mentioned. In this study, the neutron beam was generated by irradiating a proton beam upon a beryllium target using an MC-50 cyclotron, and the thermal neutron flux was low. Because the high flux of thermal neutrons is usually used for 


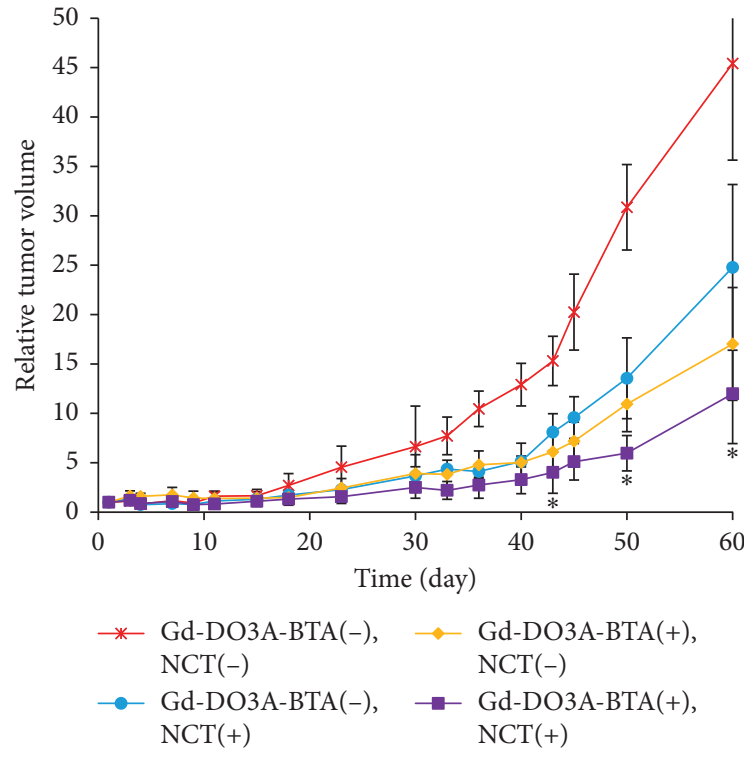

(a)

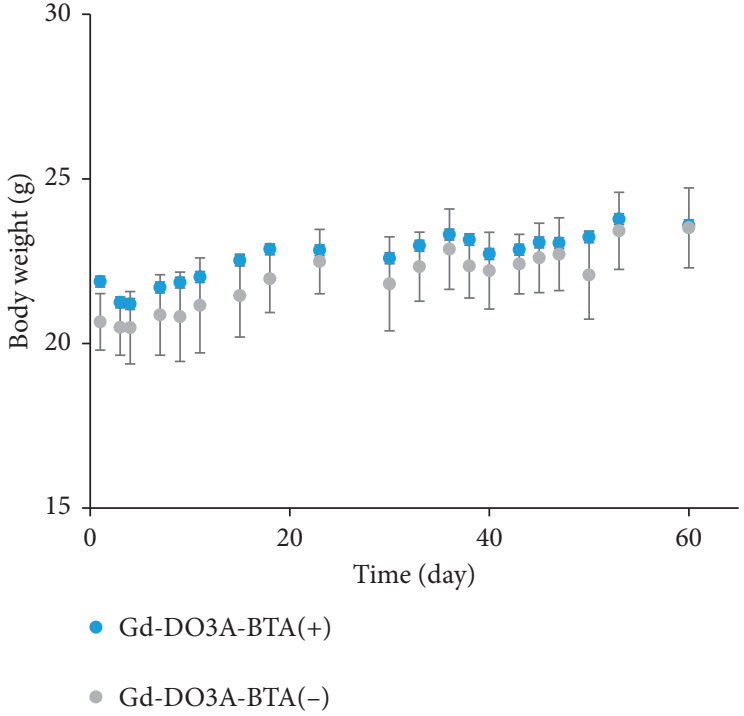

(b)

Figure 2: (a) Time-course changes in the relative tumor volume after neutron irradiation. Data are expressed as the mean \pm SD $(n=5)$, ${ }^{*} p<0.05$ compared to other groups. (b) Relative changes in the body weight of the mice.
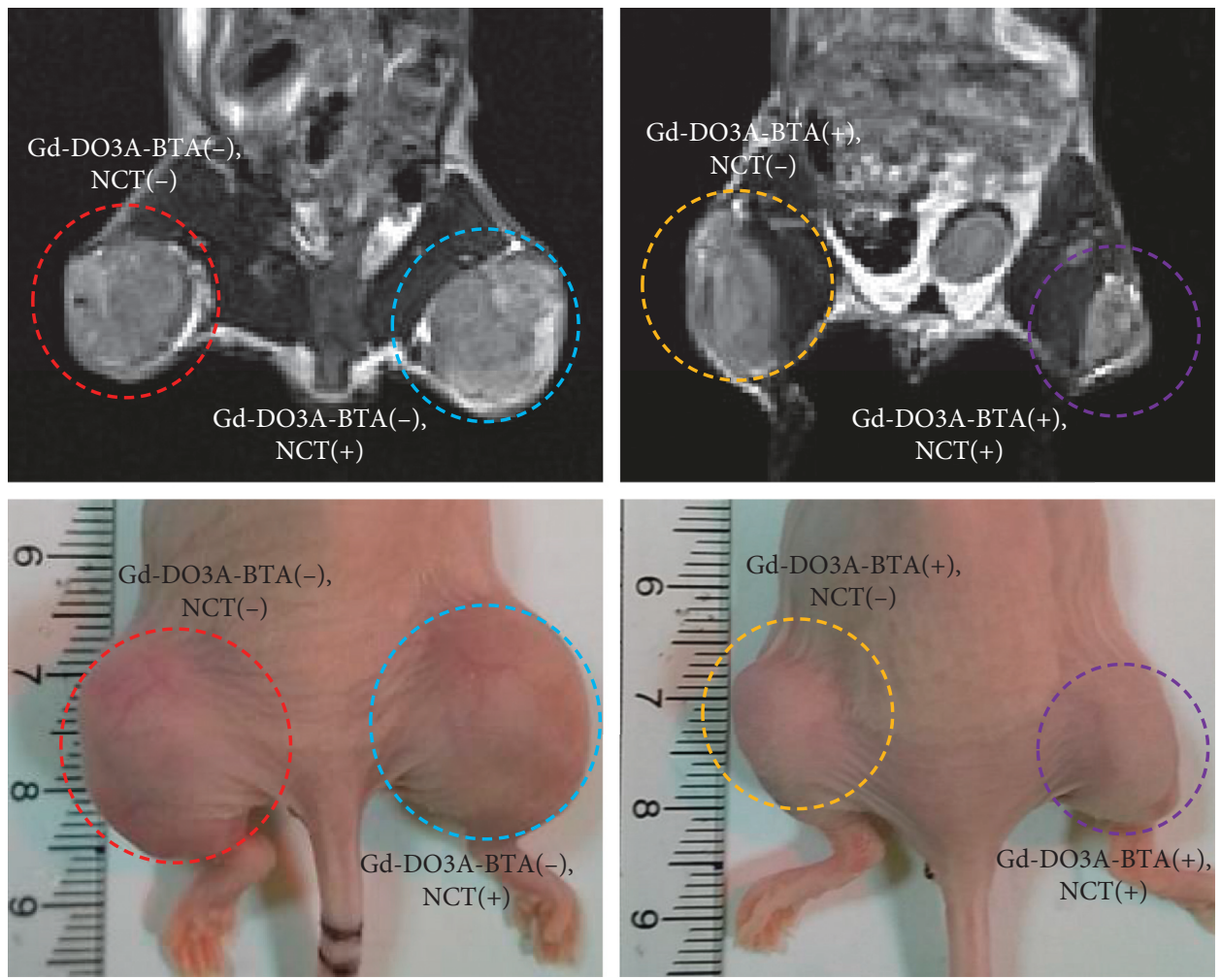

FIGURE 3: MRI (above) and morphological findings (below) of tumors at 60 days after Gd-NCT. [Gd-DO3A-BTA(+), NCT(+)], Gd-DO3ABTA administered and neutron irradiated; [Gd-DO3A-BTA(+), NCT(-)], Gd-DO3A-BTA administered and non-neutron irradiated; [Gd-DO3A-BTA(-), NCT(+)], non-Gd administered and neutron irradiated; [Gd-DO3A-BTA(-), NCT(-)], non-Gd administered and non-neutron irradiated. 

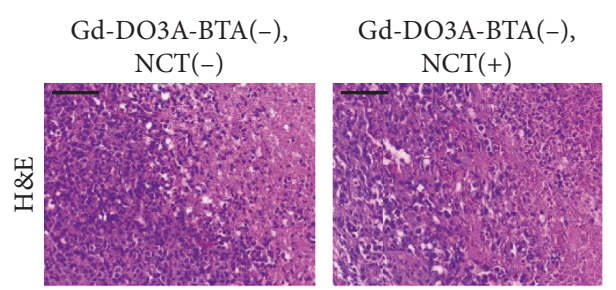

Gd-DO3A-BTA(+),

Gd-DO3A-BTA(+), NCT $(-)$ $\operatorname{NCT}(+)$
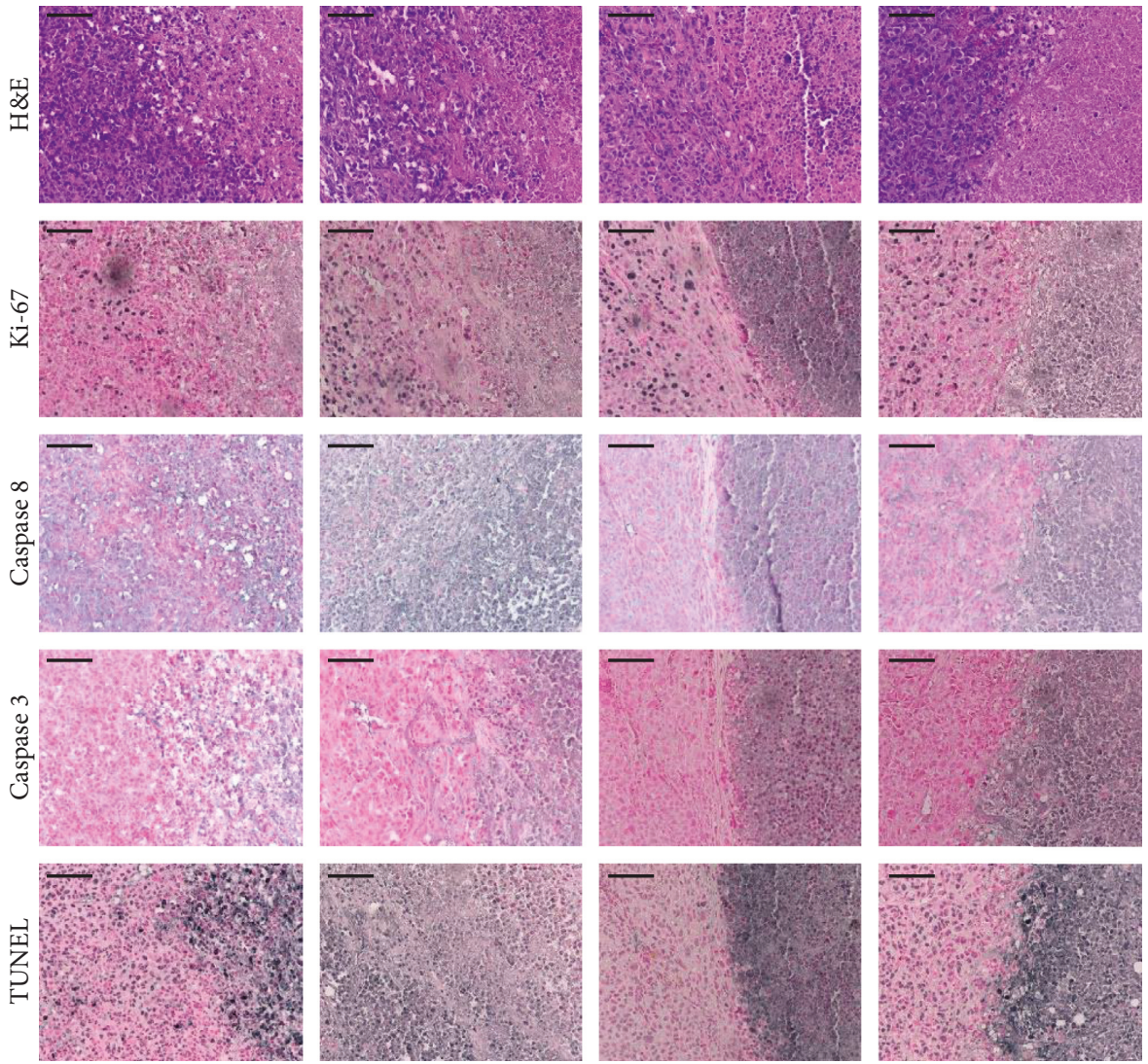

(a)

Ki-67

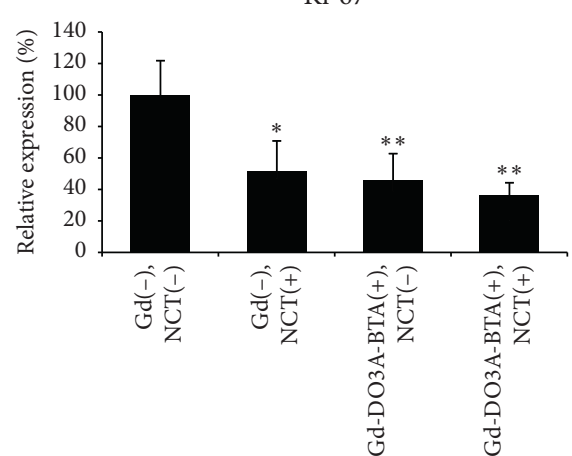

(b)

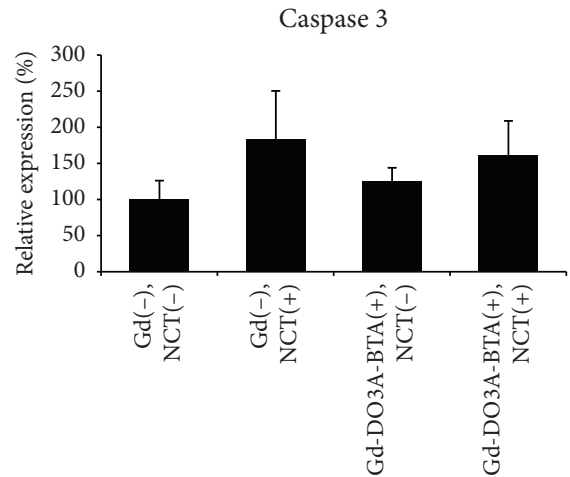

(d)

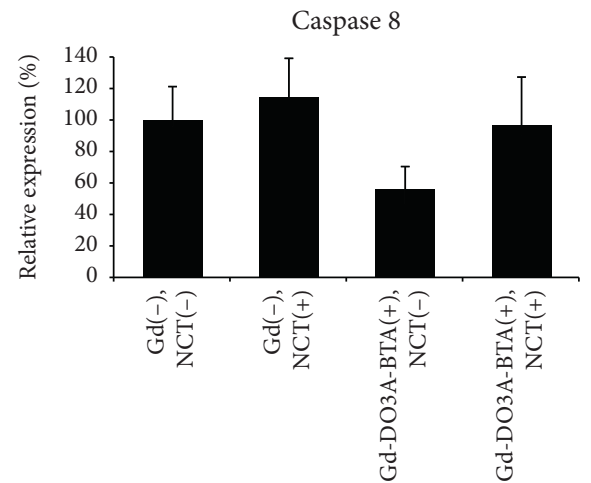

(c)

TUNEL

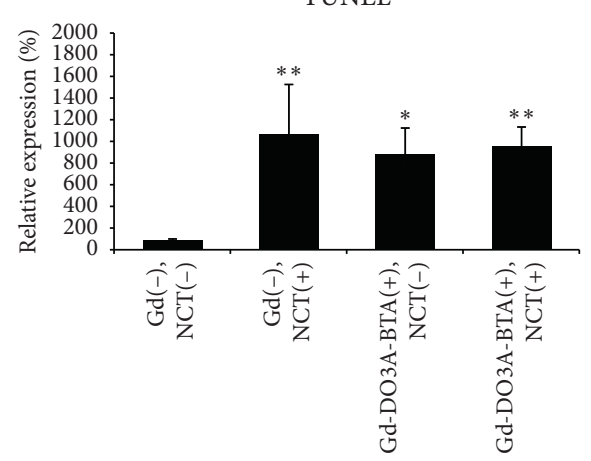

(e)

Figure 4: (a) Representative images of hematoxylin and eosin (H\&E) staining and immunohistochemical staining of Ki-67, caspase 3, caspase 8, and TUNEL in tumor samples. Graphic plots show an increase in apoptotic cells as determined by (b) Ki-67, (c) caspase 8 , (d) caspase 3, and (e) TUNEL staining in Gd-NCT. Scale bars on the images represent $100 \mu \mathrm{m} .{ }^{*} p<0.05$; ${ }^{* *} p<0.01$ compared to [Gd-DO3A-BTA(-), NCT(-)]. 
NCT, it is necessary to perform NCT experiment with low energy neutron at high flux to be sure that the effect shown in the current study is due to NCT.

\section{Conclusions}

The present study presented the potential for Gd-NCT using low molecular weight Gd chelate as a new combinatorial chemo-NCT approach in cancer treatment. Despite the simple chemical structure of the metal complex, Gd-DO3ABTA shows a high therapeutic effect when used in Gd-NCT against solid tumors. The high accumulation of Gd-DO3ABTA in tumor tissues effectively damaged the tumor cells. Furthermore, as an MR contrast agent, Gd-DO3A-BTA can guide NCT and monitor tumor growth via MR imaging.

\section{Materials and Methods}

5.1. General. The Gd-DO3A-BTA (nonenriched Gd) was prepared as described previously [10, 21]. All animal experiments were conducted in compliance with the Guidelines for the Care and Use of Research Animals under protocols approved by the Korea Institute of Radiological and Medical Sciences (KIRAMS) Animal Studies Committee. The neutron beam irradiations including low content thermal neutrons were performed using an MC-50 cyclotron (Scanditronix, Sweden, 1985). When the neutron beam was generated by irradiating a proton beam $(20 \mu \mathrm{A}, 35 \mathrm{MeV})$ upon a beryllium target of $15 \mathrm{~mm}$ thickness, the following results were previously reported: the thermal neutron $(0.24 \%$ of total neutrons) flux was approximately $1.94 \times$ $10^{4} \mathrm{n} / \mathrm{cm}^{2} \cdot \mathrm{sec}$ and the cross section was $13.79 \pm 0.45$ barn (a). At that time, the absorbed dose of neutron beams was 9.36-8.69 cGy/min (including the gamma ray of $1.42-$ $1.57 \mathrm{cGy} / \mathrm{min}$ ) at the depth of $15-30 \mathrm{~mm}$ with the field size of $26 \times 26 \mathrm{~cm}^{2}$ (b). The irradiation time of neutron beams in our studies was typically performed as the standard point of approximately $1 \mathrm{~Gy} / 12 \mathrm{~min}[32,33]$.

5.2. Tumor Model. The human breast adenocarcinoma cancer cell line MDA-MB-231 (ATCC CRM-HTB-26) was purchased from the American Type Culture Collection (ATCC). MDA-MB-231 is a highly aggressive, invasive, and poorly differentiated triple-negative breast cancer (TNBC; estrogen receptor (ER), progesterone receptor (PR), and HER2 (human epidermal growth factor receptor 2)) cell line. The cells were maintained in RPMI-1640 containing 10\% fetal bovine serum (FBS) and $1 \%$ antibiotics and were grown in a humidified incubator at $37^{\circ} \mathrm{C}$ and $5 \% \mathrm{CO}_{2}$. MDA-MB231 tumor cells $\left(1 \times 10^{8}\right.$ cells $\left.\cdot \mathrm{mL}^{-1}\right)$ suspended in RPMI- 1640 medium without FBS, and antibiotics were injected into the subcutaneous tissue (sc) of female BALB/c nude mice (aged 6 weeks, $18-25 \mathrm{~g}$ of body weight) in both legs. One week after tumor cell implantation, the mice were divided into two groups ( $n=5$ /group). To compare the effect of chemotherapy and NCT on solid tumor, the mice were administered (A) none or (B) Gd-DO3A-BTA $(0.1 \mathrm{mmol} / \mathrm{kg})$ intravenously through the tail vein. Only the right-sided tumors were irradiated by neutrons.
5.3. In Vivo MR Imaging. The mice were anesthetized with $1.5 \%$ isoflurane in oxygen. Tumor measurements were made before and after injection of $0.1 \mathrm{mmol} \cdot \mathrm{Gd} / \mathrm{kg}$ via the tail vein. MR images were taken with a $3 \mathrm{~T}$ MR unit (Magnetom Tim Trio, Siemens Medical solution, Erlangen, Germany) using an animal coil. The T1-weighted fast spin-echo imaging was performed under the following conditions: repetition time $=$ $9.9 \mathrm{~ms}$; echo time $=3.2 \mathrm{~ms} ; 10 \mathrm{~mm}$ field of view; $256 \times 256$ matrix size; $1 \mathrm{~mm}$ slice thickness; and average $=3$.

The contrast to noise ratio (CNR) was defined as the difference in signal-to-noise ratio (SNR) between adjacent anatomic structures.

$$
\mathrm{CNR}=\mathrm{SNR}_{\text {post }}-\mathrm{SNR}_{\text {pre }}
$$

5.4. In Vivo Gd-NCT. Gd-DO3A-BTA was administered intravenously as a bolus $(0.1 \mathrm{mmol} / \mathrm{kg})$ into the tail vein of female mice with MDA-MB-231 tumors. After $6 \mathrm{~h}$, the mice were locally irradiated on one side with a 0.3 Gy neutron beam, while the other side was shielded using Teflon as shown. Figure 5 shows the overall experimental scheme for Gd-NCT. The tumor size was measured before and after irradiation, and the volume $(V)$ was calculated using the following equation:

$$
V=\frac{a \times b^{2}}{2},
$$

where $a$ and $b$ are the major and minor axes of the tumor measured by a caliper.

5.5. Tissue Preparation and Histological Evaluation. The animals were sacrificed after 60 days, and tumor tissues were obtained. The samples were fixed in $10 \%$ neutral buffered formalin, processed, and embedded in paraffin, according to standard procedures. The sections were then stained with hematoxylin and eosin (H\&E) using an automated processor (Tissue-Tek, PrismaE2, Sakura, Japan). The images were acquired using a digital scanner (Panoramic MIDI, 3D HISTECH Ltd, Hungary).

5.6. Immunohistochemical Analysis. The tumor samples were serially sectioned ( $4 \mu \mathrm{m}$ sections), deparaffinized, and rehydrated. The sections were incubated in $0.3 \%$ hydrogen peroxide to quench the endogenous peroxidase after antigen retrieval by heating in $10 \mathrm{mM}$ citrate buffer ( $\mathrm{pH} \mathrm{6.0)}$. The immunohistochemistry was performed according to the avidin-biotinylated-HRP complex $(\mathrm{ABC})$ method using the Vectastain Elite ABC kit (Vector Laboratories, Burlingame, CA, USA). The primary antibodies used were Ki-67 and caspase 8 (both 1:100, Abcam, Cambridge, MA, USA) and caspase 3 ( $1: 100$, Santa Cruz, Santa Cruz, CA, USA). Apoptosis was assessed by terminal deoxynucleotidyl transferase-mediated dUTP nick-end labeling (TUNEL; Roche Diagnostics, Indianapolis, IN, USA) according to the manufacturer's instructions. All immunohistochemistry reactions and TUNEL staining were visualized by Vector SG (Vector Laboratories) and counterstained with fast nuclear 


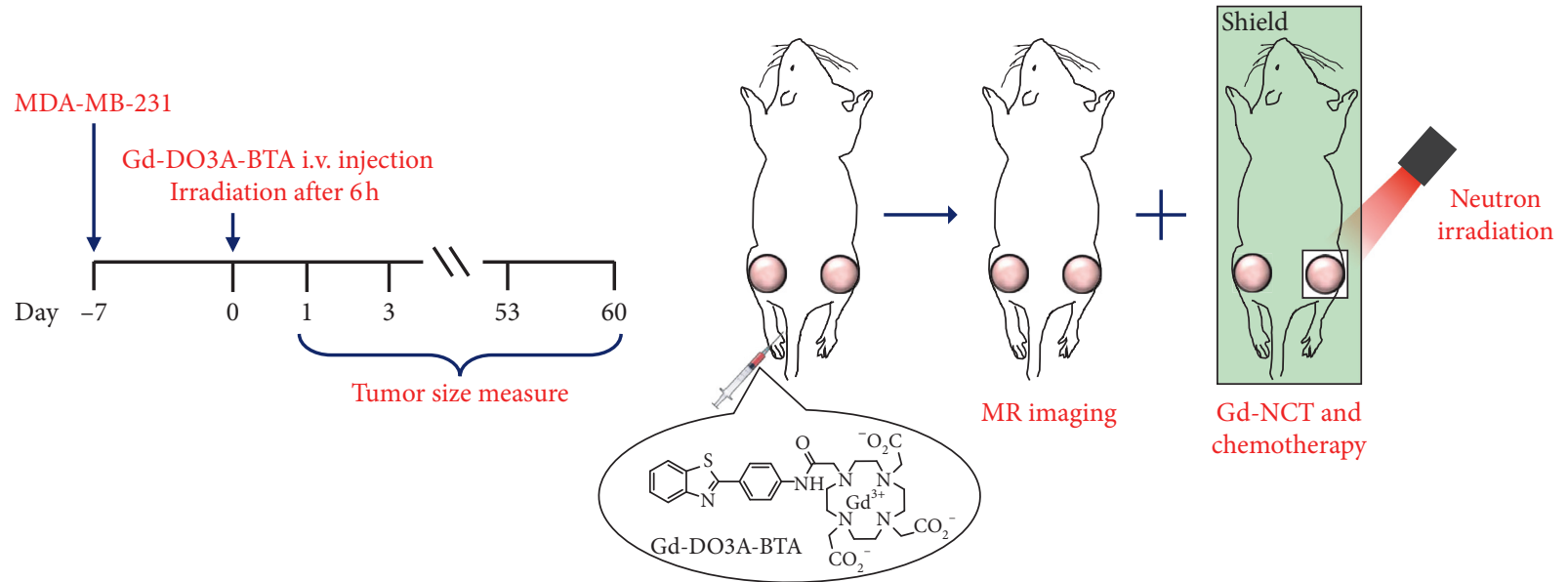

FIGURE 5: Schematic illustration of in vivo Gd-NCT. Except for the right tumor region irradiated by neutrons, the whole body was shielded by Teflon.

solutions (Vector Laboratories). Staining was quantified using ImageJ image analysis software (NIH, Bethesda, MD, USA).

5.7. Statistical Analysis. Data in the manuscript are expressed as the mean and standard deviation (SD), and the significance of the results was analyzed by Student's $t$-test. $p<0.05$ was considered statistically significant.

\section{Data Availability}

The data used to support the findings of this study are included within the article.

\section{Conflicts of Interest}

The authors declare that they have no conflicts of interest.

\section{Acknowledgments}

This study was supported by a grant from the Korea Institute of Radiological and Medical Sciences (KIRAMS) funded by the Ministry of Science, ICT and Future Planning, Republic of Korea. (no. 50532-2018). This work was also supported by the Basic Research Laboratory (BRL) Program (2013R1A4A1069507) and by the Basic Science Research Program (2017R1A2B3003214) through the National Research Foundation funded by the Ministry of Science, ICT and Future Planning.

\section{References}

[1] R. M. Brugger and J. A. Shih, "Evaluation of gadolinium-157 as a neutron capture therapy agent," Strahlentherapie und Onkologie, vol. 165, no. 2-3, pp. 153-156, 1989.

[2] C. Salt, A. J. Lennox, M. Takagaki, J. A. Maguire, and N. S. Hosmane, "Boron and gadolinium neutron capture therapy," Russian Chemical Bulletin, vol. 53, no. 9, pp. 1871$1888,2004$.

[3] P. Mi, H. Yanagie, N. Dewi et al., "Block copolymer-boron cluster conjugate for effective boron neutron capture therapy of solid tumors," Journal of Controlled Release, vol. 254, pp. 1-9, 2017.

[4] R. F. Barth, M. G. H. Vicente, O. K. Harling et al., "Current status of boron neutron capture therapy of high grade gliomas and recurrent head and neck cancer," Radiation Oncology, vol. 7, no. 1, pp. 146-167, 2012.

[5] R. F. Barth, Z. Zhang, and T. Liu, "A realistic appraisal of boron neutron capture therapy as a cancer treatment modality," Cancer Communications, vol. 38, no. 1, pp. 1-7, 2018.

[6] Z. Zhou and Z.-R. Lu, "Gadolinium-based contrast agents for magnetic resonance cancer imaging," Wiley Interdisciplinary Reviews: Nanomedicine and Nanobiotechnology, vol. 5, no. 1, pp. 1-18, 2013.

[7] H. Tokumitsu, H. Ichikawa, and Y. Fukumori, "Chitosangadopentetic acid complex nanoparticles for gadolinium neutron-capture therapy of cancer: preparation by novel emulsion-droplet coalescence technique and characterization," Pharmaceutical Research, vol. 16, no. 12, pp. 1830-1835, 1999.

[8] T. Zhang, A. Matsumura, T. Yamamoto, F. Yoshida, and T. Nose, "Comparison of gadobenate dimeglumine and gadopentetate Dimeglumine: a study of MR imaging and inductively coupled plasma atomic emission spectroscopy in rat brain tumors," American Journal of Neuroradiology, vol. 23, no. 1, pp. 15-18, 2002.

[9] H. K. Kim, M. K. Kang, K. H. Jung et al., "Gadolinium complex of DO3A-benzothiazole aniline (BTA) conjugate as a theranostic agent," Journal of Medicinal Chemistry, vol. 56, no. 20, pp. 8104-8111, 2013.

[10] N. Dewi, H. Yanagie, H. Zhu et al., "Tumor growth suppression by gadolinium-neutron capture therapy using gadolinium-entrapped liposome as gadolinium delivery agent," Biomedicine and Pharmacotherapy, vol. 67, no. 6, pp. 451-457, 2013.

[11] U. M. Le and Z. Cui, "Long-circulating gadoliniumencapsulated liposomes for potential application in tumor neutron capture therapy," International Journal of Pharmaceutics, vol. 312, no. 1-2, pp. 105-112, 2006.

[12] U. M. Le and Z. Cui, "Biodistribution and tumoraccumulation of gadolinium (Gd) encapsulated in longcirculating liposomes in tumor-bearing mice for potential neutron capture therapy," International Journal of Pharmaceutics, vol. 320, no. 1-2, pp. 96-103, 2006. 
[13] M. M. Iyamoto, K. H. Irano, H. I. Chikawa, Y. F. Ukumori, and Y. A. Kine, "Biodistribution of gadolinium incorporated in lipid emulsions intraperitoneally administered for neutroncapture therapy with tumor-bearing hamsters," Biological and Pharmaceutical Bulletin, vol. 22, no. 12, pp. 1331-1340, 1999.

[14] P. Mi, N. Dewi, H. Yanagie et al., "Hybrid calcium phosphatepolymeric micelles incorporating gadolinium chelates for imaging-guided gadolinium neutron capture tumor therapy," ACS Nano, vol. 9, no. 6, pp. 5913-5921, 2015.

[15] N. Dewi, P. Mi, H. Yanagie, and Y. Sakurai, "In vivo evaluation of neutron capture therapy effectivity using calcium phosphate-based nanoparticles as Gd-DTPA delivery agent," Journal of Cancer Research and Clinical Oncology, vol. 142, no. 4, pp. 767-775, 2016.

[16] H. Ichikawa, T. Uneme, T. Andoh, Y. Arita, and T. Fujimoto, "Gadolinium-loaded chitosan nanoparticles for neutroncapture therapy: influence of micrometric properties of the nanoparticles on tumor-killing effect," Applied Radiation and Isotopes, vol. 88, pp. 109-113, 2014.

[17] H. Tokumitsu, J. Hiratsuka, Y. Sakurai, T. Kobayashi, H. Ichikawa, and Y. Fukumori, "Gadolinium neutron-capture therapy using novel gadopentetic acid-chitosan complex nanoparticles: in vivo growth suppression of experimental melanoma solid tumor," Cancer Letters, vol. 150, no. 2, pp. 177-182, 2000.

[18] A. Narmani, B. Farhood, H. Haghi-aminjan, and T. Mortezazadeh, "Gadolinium nanoparticles as diagnostic and therapeutic agents: their delivery systems in magnetic resonance imaging and neutron capture therapy," Journal of Drug Delivery Science and Technology, vol. 44, pp. 457-466, 2018.

[19] G. de Stasio, P. Casalbore, R. Pallini et al., "Gadolinium in human glioblastoma cells for gadolinium neutron capture therapy," Cancer research, vol. 61, no. 10, pp. 4272-4277, 2001.

[20] A. Kamal, M. A. H. Syed, and S. M. Mohammed, "Therapeutic potential of benzothiazoles: a patent review (2010-2014)," Expert Opinion on Therapeutic Patents, vol. 25, no. 3, pp. 335-349, 2015.

[21] K. H. Jung, S. H. Kang, M. K. Kang et al., "Synthesis and structure-activity relationships of gadolinium complexes of DO3A-benzothiazole conjugates as potential theranostic agents," European Journal of Inorganic Chemistry, vol. 2015, no. 4, pp. 599-604, 2015.

[22] P. C. Burge, F. Stephen Vogel, S. B. Green, and T. A. Strike, "Glioblastoma multiforme and anaplastic astrocytoma pathologic criteria and prognostic implications," Cancer, vol. 56, no. 5, pp. 1106-1111, 1985.

[23] J. M. Bruner, "Neuropathology of malignant gliomas," Seminars in Oncology, vol. 21, no. 2, pp. 126-138, 1994.

[24] B. A. Edadah and A. I. Faden, "Caspase pathways, neuronal apoptosis, and CNS injury," Jounal of Neurotauma, vol. 17, no. 10 , pp. $811-829,2000$.

[25] J. Shih and R. Brugger, "Gadolinium as a neutron capture therapy agent," Medical Physics, vol. 19, no. 3, pp. 733-744, 1992.

[26] T. Watanabe, H. Ichikawa, and Y. Fukumori, "Tumor accumulation of gadolinium in lipid-nanoparticles intravenously injected for neutron-capture therapy of cancer," European Journal of Pharmaceutics and Biopharmaceutics, vol. 54, no. 2, pp. 119-124, 2002.

[27] F. Shikata, H. Tokumitsu, H. Ichikawa, and Y. Fukumori, "In vitro cellular accumulation of gadolinium incorporated into chitosan nanoparticles designed for neutron-capture therapy of cancer," European Journal of Pharmaceutics and Biopharmaceutics, vol. 53, no. 1, pp. 57-63, 2002.

[28] D. Alberti, N. Protti, M. Franck et al., "Theranostic nanoparticles loaded with imaging probes and rubrocurcumin for combined cancer therapy by folate receptor targeting," ChemMedChem, vol. 12, no. 7, pp. 502-509, 2017.

[29] A. M. Dierling, B. R. Sloat, and Z. Cui, "Gadolinium incorporated reconstituted chylomicron emulsion for potential application in tumor neutron capture therapy," European Journal of Pharmaceutics and Biopharmaceutics, vol. 62, no. 3, pp. 275-281, 2006.

[30] K. J. Ono, H. I. Chikawa, K. F. Ujioka, Y. F. Ukumori, and Y. A. Kine, "Preparation of lecithin microcapsules by a dilution method using the wurster process for intraarterial administration in gadolinium neutron capture therapy," Chemical and Pharmaceutical Bulletin, vol. 47, no. 1, pp. 54-63, 1999.

[31] M. R. Longmire, M. Ogawa, P. L. Choyke, and H. Kobayashi, "Biologically optimized nanosized molecules and particles: more than just size," Bioconjugate Chemistry, vol. 22, no. 6, pp. 993-1000, 2011.

[32] S. Park and N. H. Jang, "Estimation and calibration of thermal neutron flux for neutron activation analysis," Bulletin of the Korean Chemical Society, vol. 27, no. 12, pp. 2061-2063, 2006.

[33] K.-Y. Eom, H.-G. Wu, H. J. Park et al., "Evaluation of biological Characteristics of neutron beam generated from MC50 cyclotron," Journal of the Korean Society for Therapeutic Radiology and Oncology, vol. 24, no. 4, pp. 280-284, 2006. 


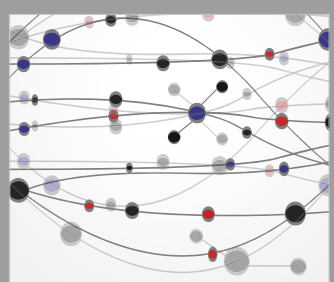

The Scientific World Journal
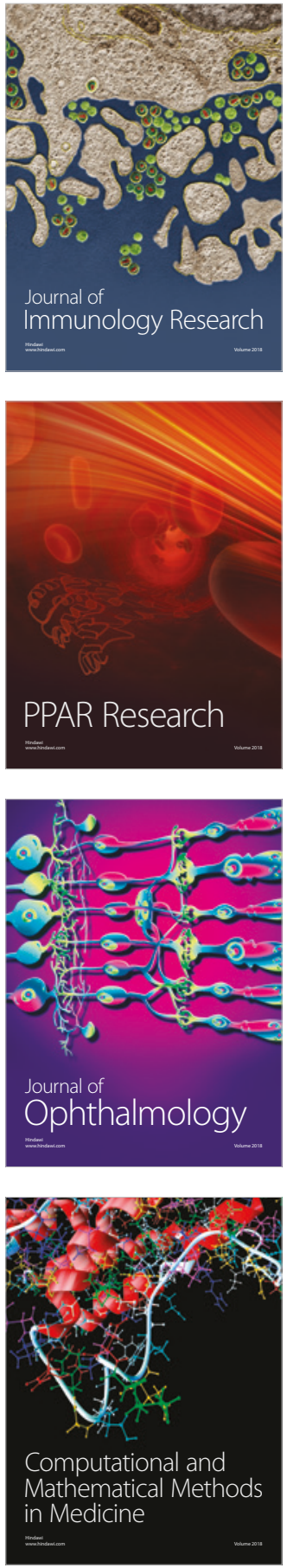

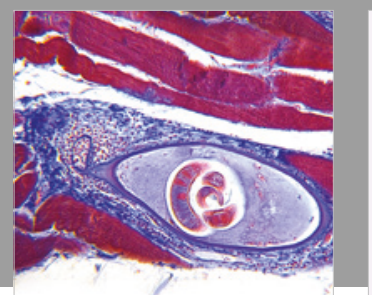

Gastroenterology Research and Practice

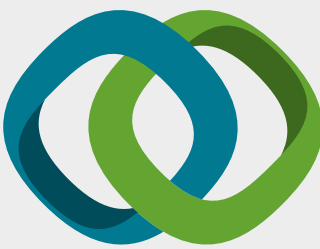

\section{Hindawi}

Submit your manuscripts at

www.hindawi.com
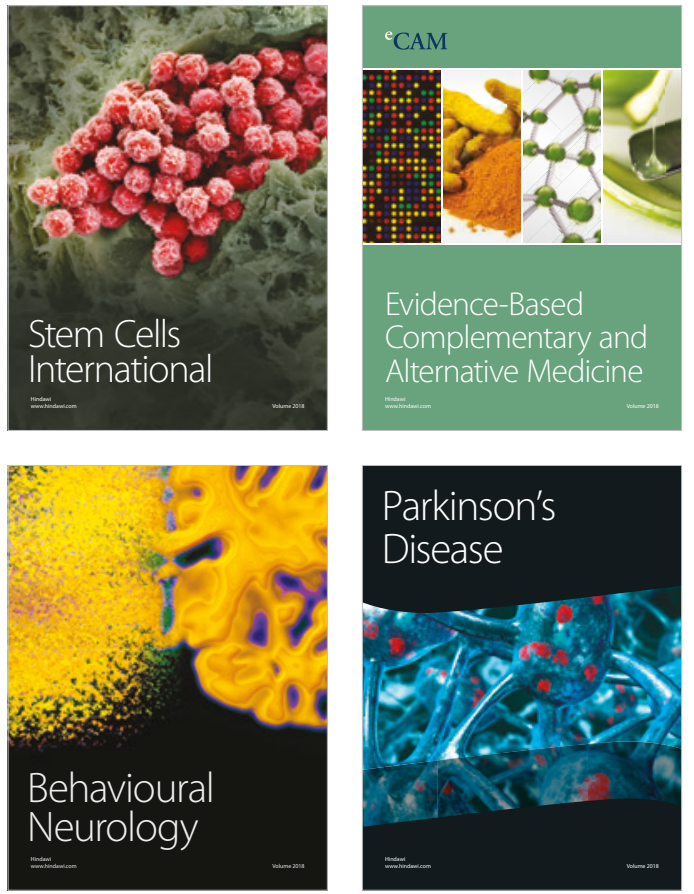

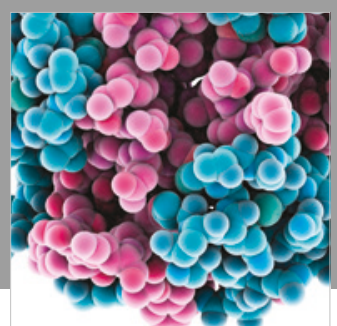

ournal of

Diabetes Research

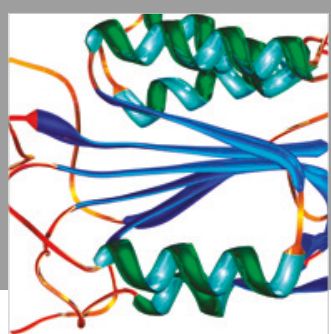

Disease Markers
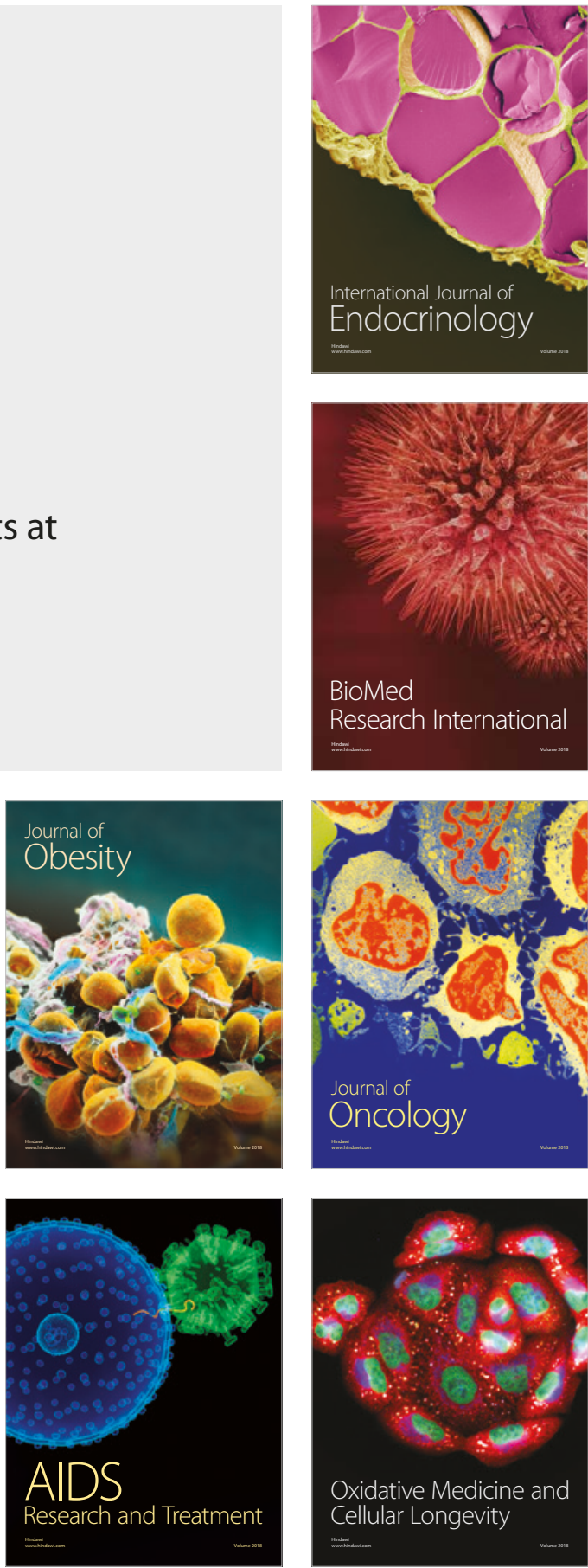